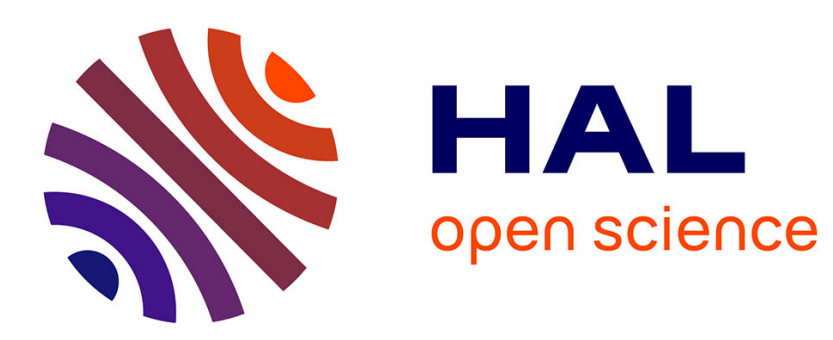

\title{
Dynamic environment modeling with gridmap: a multiple-object tracking application
}

Cheng Chen, Christopher Tay, Kamel Mekhnacha, Christian Laugier

\section{To cite this version:}

Cheng Chen, Christopher Tay, Kamel Mekhnacha, Christian Laugier. Dynamic environment modeling with gridmap: a multiple-object tracking application. Proc. of the Int. Conf. on Control, Automation, Robotics and Vision, Dec 2006, Singapour, Singapore. inria-00182007

\section{HAL Id: inria-00182007 https://hal.inria.fr/inria-00182007}

Submitted on 24 Oct 2007

HAL is a multi-disciplinary open access archive for the deposit and dissemination of scientific research documents, whether they are published or not. The documents may come from teaching and research institutions in France or abroad, or from public or private research centers.
L'archive ouverte pluridisciplinaire HAL, est destinée au dépôt et à la diffusion de documents scientifiques de niveau recherche, publiés ou non, émanant des établissements d'enseignement et de recherche français ou étrangers, des laboratoires publics ou privés. 


\title{
Dynamic environment modeling with gridmap: a multiple-object tracking application
}

\author{
C. Chen, C. Tay, K. Mekhnacha, and C. Laugier \\ INRIA Rhone-Alpes \\ 655 avenue de l'Europe - 38334 St Ismier Cedex, France \\ Email: \{ccheng; tay; christian.laugier\}@inrialpes.fr
}

\begin{abstract}
The Bayesian occupancy filter (BOF) [1] has achieved promising results in the object tracking applications. This paper presents a new development of BOF which inherits original BOF's advantages in handling occlusion and representing objects' shape. Meanwhile, the new formulation has significantly reduced original BOF's complexities and can be run in realtime. In Bayesian occupancy filter, the environment is finely divided into 2-dimensional grids. Different from conventional occupancy gridmaps, in BOF, each grid has both static (occupancy) and dynamic (velocity) characteristics. In the new proposed BOF, the velocity of each cell is modeled as a distribution. The distribution for each cell occupancy can therefore be inferred using a filtering mechanism. Like the original $\mathrm{BOF}$, no representation of objects exists in the BOF gridmap. However, there are often applications which require the definition and tracking at the object level. In the post-processing, a segmentation algorithm is implemented to extract the objects from BOF estimation. Thereafter, standard target tracking methods are employed to further analyze each object's motion. Experiments using data from an indoor human tracking application demonstrate that our approach yields satisfactory results even when serious occlusions exist.
\end{abstract}

\section{INTRODUCTION}

Perception and reasoning with dynamic environments is pertinent for mobile robotics and still constitutes one of the major challenges. To work in these environments, the mobile robot needs to perceive through its sensors, where measurements are uncertain. Uncertainty is often treated within the estimation framework. Such an approach enables the mobile robot to model the dynamic environment and follow the evolution of its environment. With an internal representation of the environment, the robot is thus able to perform reasoning and predictions in order to accomplish its tasks successfully. Systems for tracking the evolution of the environment has been traditionally a major component in robotics. Industries are now beginning to express their interests in such technologies. One particular example is the application within the automotive industry for adaptive cruise control [1] where the challenge is to reduce road accidents, thanks to better collision detection systems. The major requirement of such a system is a robust tracking system. Most of the existing target tracking algorithms uses an object-based representation of the environment. However, these existing techniques have to take into account explicitly data association and occlusion. In view of these problems, the bayesian occupancy filter (BOF) [2] has been proposed.
The advantages of the BOF are:

- Uncertainty taken into account explicitly thanks to the probabilistic reasoning paradigm, which is becoming a key paradigm in robotics: various approaches based on this paradigm have already been successfully used to address several robotic problems, such as CAD modelling [3] or map building and localization (SLAM) [4], [5].

- The data association problem is postponed by reasoning on a probabilistic grid representation of the dynamic environment. In such a model, concepts such as objects or tracks do not exist; they are replaced by more useful properties such as occupancy or risk, which are directly estimated for each cell of the grid using both sensor observations and some prior knowledge. For example, to calculate the risk of collision for a mobile robot, the only properties required are the distribution on occupancy and velocities for each cell in the grid with no strict obligations to deal with data association. Furthermore, in estimating the occupancy probability the hidden parts of the environments can also be explicitly characterized, thanks to an adequate sensor model.

- The object model problem is nonexistent because there are only cells in the environment state and that each sensor measurement changes the state of each cell. The fact that different kinds of object produces different kinds of measures are handled naturally by the cell space discretization.

- The dynamics of the environment and its robustness relative to object occlusions is addressed using a novel two-step mechanism which permits taking the sensor observations history and the temporal consistency of the scene into account. This mechanism estimates, at each time step, the state of the occupancy grid by combining a prediction step (history) and an estimation step (incorporating new measurements). This approach is derived from the Bayes filters approach [6]; which explains why the filter is called the Bayesian Occupancy Filter (BOF).

- The Bayesian Occupancy Filter has been designed in order to be highly parallelisable. A hardware implementation on a dedicated chip is possible, which will lead to an efficient representation of the environment of a mobile robot. 


\section{A. Contributions}

Previous experiments based on the BOF techniques relied on the assumption of a constant velocity and the problem of velocity estimation in this context has not been addressed. In particular the assumption that there could only be one object with one velocity in each cell was not part of the previous model. In this paper, a representation that has one probability distribution over velocities for each occupancy cell is presented. This model is very similar in concept to optical flow, but with occupancy considerations rather than intensity. The general principle for the estimation of occupancy grids will be to include the velocity estimation in the prediction estimation loop of the classical BOF approach. For each grid in the BOF, the set of velocities that brings a set of corresponding cells in the previous time step to the current grid will be considered. The resulting distribution on the velocity of the current grid is updated by conditioning on the incoming velocities with respect to the current grid and on the observations.

This paper brings an amelioration in these aspects and thus presents an integrated approach to performing not only the occupancy states of the grids but the distribution on grid velocity as well. To avoid confusion, all ideas presented applies to the currently proposed formulation of BOF unless explicitly stated. The current paper is organized as follow:

- in section II, related work to multiple target tracking systems and occupancy grids are presented.

- in section III, the fundamental concept of bayesian filtering and the new filtering equations in the grids are presented.

- Section IV presents the object-level abstraction based on BOF estimation. A nearest neighbor join probabilistic data association algorithm is also developed to track the extracted objects.

- The paper finishes off with a presentation of results followed by a conclusion.

\section{RELATED WORK}

\section{A. Multi-Target Tracking}

The aim of multi-target tracking is to estimate at each time step, the dynamics of each moving object observed by the sensors. Such an estimation is required due to uncertainty in observations i.e. sensor data. The estimation of the dynamics is performed, in a manner that is as robust as possible, after observations are obtained from the sensors. The main difficulty of multi-target tracking is known as the Data Association problem. It includes observation-to-track association and track management problems. The goal of observation-to-track association is to decide whether a new sensor observation corresponds to an existing track. Track management includes deciding whether existing tracks should be maintained, deleted, or if new tracks should be created. Numerous methods exist to perform data association [7], [8]. The reader is referred to [9] for a complete review of the existing tracking methods with one or more sensors.
Urban traffic scenarios are still a challenge in multitarget tracking area: the traditional data association problem is intractable in situations involving numerous appearances, disappearances and occlusions of a large number of rapidly manoeuvring targets.

In [10], a classical Multiple Hypothesis Tracking technique is used to track moving objects while stationary objects are used for SLAM. Unfortunately, the authors did not explicitly address the problem of the interaction between tracked and stationary objects, e.g. when a pedestrian is temporary hidden by a parked car. One of the aims of the BOF is to overcome such a problem.

\section{B. Grid Representation of the Environment}

The occupancy grids framework [11], [12] is a classical way to describe the environment of a mobile robot. It has been extensively used for static indoor mapping using a 2-dimensional grid. The goal is to compute from the sensor observations the probability of each cell being occupied or empty. To avoid a combinatorial explosion of grid configuration, the cell states are estimated as independent random variables.

More recently, occupancy grids have been adapted to track multiple moving objects [13]. In this approach, spatiotemporal clustering applied to temporal maps is used to perform motion detection and tracking. A major drawback of this work, relative to the ADAS context, is that a moving object may be lost due to occlusion effects.

\section{BAyESIAN OCCUPANCY FILTER}

\section{A. Grid Based Bayesian Filtering}

The sensor observation history should be taken into account in order to be able to make robust estimations in changing environments (i.e. in order to be able to process temporary objects occlusions and detection problems). Our approach for solving this problem is to make use of an appropriate Bayesian filtering technique called the Bayesian Occupancy Filter (BOF).

Bayes filters [6] address the general problem of recursively estimating the probability distribution, $P\left(X^{k} \mid Z^{k}\right)$, of the state of a system conditioned on its observation. This expression is also known as the posterior distribution. In general, this estimation is done in two stages: prediction and estimation.

The goal of the prediction stage is to compute an a priori estimate of the target's state known as the prior distribution.

The goal of the estimation stage is to compute the posterior distribution by using the estimate from the previous time step with the current measurement of the sensor.

Exact solutions to this recursive propagation of the posterior density do exist in a restrictive set of cases. In particular, the Kalman filter [14] is an optimal solution when the measurement and state transition model are linear with additive gaussian noise. But in general, solutions cannot be determined analytically, and hence an approximate solution has to be computed.

In the case of the BOF, the state of the system is given by the occupancy state and velocity of each cell of the grid, and the 
required conditions for being able to apply an exact solution such as the Kalman filter are not always verified. In addition, the particular structure of the model (grids) and the real-time constraint coming from most practical robotic applications leads to the development of the Bayesian Occupancy Filter.

With the improved BOF formulation, it is now possible to focus the computation on the most probable velocities of grids instead of updating the grid occupancy values for every possible velocity in the standard BOF approach. Such an approach is not only more efficient computationally, but provides a more theoretically founded and systematic way of estimating grid velocities.

\section{B. Bayesian Model}

1) Probabilistic variable definitions: All the probabilistic variables below are defined within the context of a single cell $c$ of the grid. This subscript is now omitted to maintain simplicity, except where ambiguity is possible.

- $C$ is an index that identify each $2 \mathrm{D}$ cell of the grid.

- $A$ is an index that identify each possible antecedent of the cell $c$ over all the cells in the 2D grid.

- $Z_{t} \in \mathcal{Z}$ where $Z_{t}$ is the random variable of the sensor measurement relative to the cell $c$.

- $V \in \mathcal{V}=\left\{v_{1}, \ldots, v_{n}\right\}$ where $V$ is the random variable of the velocities for the cell $c$ and its possible values are discretized in $n$ cases.

- $O, O^{-1} \in \mathcal{O} \equiv\{o c c, e m p\}$ where $O$ represents the random variable of the state of $c$ being either "occupied" or "empty". $O^{-1}$ represents the random variable of the state of an antecedent cell of $c$ through the possible motion through $c$. For a given velocity $v_{k}=\left(v_{x}, v_{y}\right)$ and a given time step $\delta t$, it is possible to define an antecedent for $c=(x, y)$ as $c^{-k}=\left(x-v_{x} \delta t, y-v_{y} \delta t\right)$.

2) Joint distributions: The following expression is the decomposition of the joint distribution of the all the relevant variables according to bayes'rule and independency assumptions.

$$
\begin{aligned}
& P\left(C, A, Z, O, O^{-1}, V\right)=P(A) P(V \mid A) P(C \mid V, A) \\
& P\left(O^{-1} \mid A\right) P\left(O \mid O^{-1}\right) P(Z \mid O, V, C)
\end{aligned}
$$

The semantics of each distribution in the decomposition are interpreted as follows:

- $P(A)$ is the distribution over all the possible antecedent of the cell $c$. It is chosen to be uniform because the cell is considered reachable from all the antecedents with equal probability.

- $P(V \mid A)$ is the distribution over all the possible velocities of a certain antecedent of the cell $c$, its parametric form is in the form of a histogram.

- $P(C \mid V, A)$ is a distribution that explains if $c$ is reachable from $[A=a]$ with the velocity $[V=v]$. It is a dirac with value equal to one if and only if $c_{x}=a_{x}+v_{x} \delta t$ and $c_{y}=a_{y}+v_{y} \delta t$ which follows a dynamic model of constant velocity.
- $P\left(O^{-1} \mid A\right)$ is the conditional distribution over the occupancy of the antecedent. It gives the probability of the possible previous step of the the current cell.

- $P\left(O \mid O^{-1}\right)$ is the conditional distribution over the occupancy of the current cell, which depends on the occupancy state of the previous cell. It is defined as a transition matrix: $T=\left[\begin{array}{cc}1-\epsilon & \epsilon \\ \epsilon & 1-\epsilon\end{array}\right]$, which allows the system to take in account the fact that the null acceleration hypothesis is an approximation; in this matrix, $\epsilon$ is a parameter representing the probability that the object in $c$ does not follow the null acceleration model.

- $P(Z \mid O, V, C)$ is the conditional distribution over the sensor measurement values. It depends of the state of the cell, the velocity of the cell and obviously the position of the cell.

3) Filtering Computation and Representation: Th aim of filtering in the BOF grid is to estimate the distribution and grid velocity for each cell of the grid. From the implementation point of view, the set of possible velocities are discretized. One way of implementating the computation of the probability distribution is in the form of histograms. The following equations displayed are based on the discrete case. Therefore, the global filtering equation can be obtained by:

$$
P(V, O \mid Z, C)=\frac{\sum_{A, O^{-1}} P\left(C, A, Z, O, O^{-1}, V\right)}{\sum_{A, O, O^{-1}, V} P\left(C, A, Z, O, O^{-1}, V\right)}(2)
$$

Which can be equivalently represented as:

$$
\begin{aligned}
& P(V, O, Z, C)=P(Z \mid O, V, C) * \\
& {\left[\sum_{A, O^{-1}} P(A) P(V \mid A) P(C \mid V, A) P\left(O^{-1} \mid A\right) P\left(O \mid O^{-1}\right)\right]}
\end{aligned}
$$

The summation in the expression represents the prediction and its multiplication with the first term, $P(Z \mid O, V, C)$, gives the estimation.

The global filtering equation (2) can actually be separated into three stages. The first being the prediction of the grid occupancy given the velocity:

$$
\begin{aligned}
\alpha\left(\text { occ }, v_{k}\right)= & \sum_{A, O^{-1}} P(A) P\left(v_{k} \mid A\right) P(C \mid A) P\left(O^{-1} \mid A\right) * \\
& P\left(\text { occ } \mid O^{-1}\right), \\
\alpha\left(e m p, v_{k}\right)= & \sum_{A, O^{-1}} P(A) P\left(v_{k} \mid A\right) P(C \mid A) P\left(O^{-1} \mid A\right) * \\
& P\left(e m p \mid O^{-1}\right) .
\end{aligned}
$$

The observation (defined for a certain velocity) can be given by:

$$
\begin{aligned}
\beta\left(o c c, v_{k}\right) & =P\left(Z \mid o c c, v_{k}\right) \alpha\left(o c c, v_{k}\right) \\
\beta\left(e m p, v_{k}\right) & =P\left(Z \mid e m p, v_{k}\right) \alpha\left(e m p, v_{k}\right) .
\end{aligned}
$$


And the likelihood of a certain velocity is given by:

$$
l\left(v_{k}\right)=\beta\left(o c c, v_{k}\right)+\beta\left(e m p, v_{k}\right) .
$$

Finally the estimation on probability of occupancy for cell $C$ with velocity $v_{k}$ is:

$$
P\left(\text { occ }, v_{k}, Z, C\right)=\frac{\beta\left(o c c, v_{k}\right)}{l\left(v_{k}\right)} .
$$

The occupancy distribution in one cell can be obtained by the marginalization over the velocities and the velocity distribution by the marginalization over the occupancy values:

$$
\begin{aligned}
& P(O \mid Z, C)=\sum_{V} P(V, O \mid Z, C), \\
& P(V \mid Z, C)=\sum_{O} P(V, O \mid Z, C) .
\end{aligned}
$$

What can be surprising is that, a tracking system will only focus on the velocity for occupied cells, that is $P(o c c, V)$ and only for cells that are high probability to be occupied.

\section{OBJECT-LEVEL POST-PROCESSING}

Although the Bayesian Occupancy Filter yields satisfactory results, in some specific applications it is often desirable to maintain the object-level abstraction for higher-level analysis. For instance, in unmanned surveillance, the number of objects (people) in the surveillance region must be reported. In this work, an optional object extraction and tracking module is included to post-process the result of BOF.

\section{A. Extract objects from BOF}

The first step is a segmentation routing which locates individual regions in the environment. BOF provides coherent estimation for the occupancy and velocity estimation, so that a naive discontinuity based routing is already sufficient to perform the segmentation. Here we employ a simplified version of the graph-based clustering scheme in [15]. The similarity between two nearby grids is computed as the inverse of the Euclidean distance between them in the joint space defined by the position, occupancy value and velocity. Grids with high similarities are grouped into the same cluster.

\section{B. Kalman filter for each object}

On the object level, we use Kalman filter (KF) to handle the movement of each occupied region. The movement is modeled to have a constant velocity, which is consistent with the BOF framework. However, different from the conventional 2D constant velocity KF, here the velocity is also observable, thanks to the speed estimation from BOF. Therefore, the state vector and observational matrix in this implementation of $\mathrm{KF}$ are written as:

$$
\overline{\mathbf{X}}=\left(\begin{array}{c}
\mathbf{x} \\
\mathbf{y} \\
\dot{\mathbf{x}} \\
\dot{\mathbf{y}}
\end{array}\right) \quad \mathbf{H}=\left(\begin{array}{cccc}
1 & 0 & 0 & 0 \\
0 & 1 & 0 & 0 \\
0 & 0 & 1 & 0 \\
0 & 0 & 0 & 1
\end{array}\right)
$$

where $\mathbf{x}$ and $\mathbf{y}$ represent the mass center of the extracted region weighted by the occupancy likelihood.

\section{NNJPDA for data association}

Conventional 'report-to-track' trackers often suffer a lot from the data association problem. In this work, since the BOF process has significantly resolved the ambiguity in the report/track assignment, in the post-processing, the data association problem can be trivially solved by joint probabilistic data association (JPDA).

JPDA is a well-established data association approach that has gained tremendous popularity in various tracking applications, including human tracking [16], air-craft tracking [], and visual tracking [17]. Its strength lies in its capability to jointly calculate the likelihood of association hypotheses, and therefore preclude potential erroneous assignments generated by greedy association algorithms (nearest neighborhood). A typical association event and the corresponding result of JPDA can be found in Fig.1 and the following table.

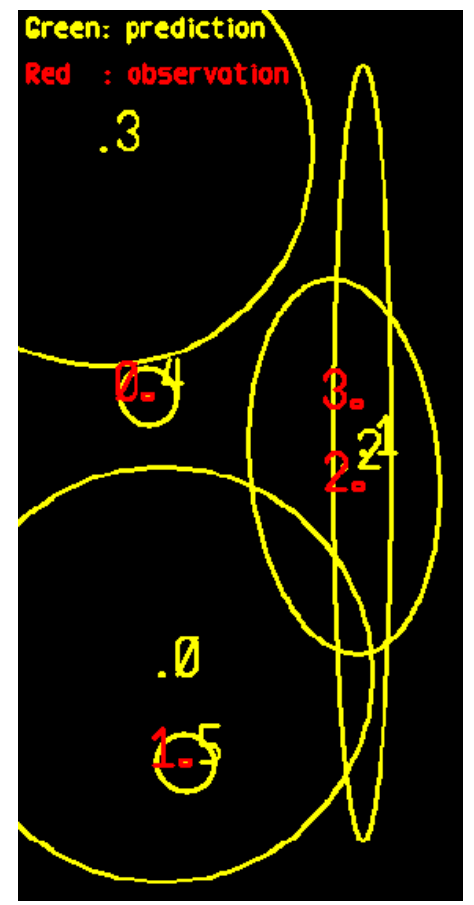

Fig. 1. An illustrative association event in JPDA. Each track's prediction state and covariance ellipse is plotted. The observations are also depicted and indexed.

TABLE I

THE LIKELIHOODS CALCULATED BY JPDA FOR REPORT-TO-TRACK ASSOCIATION CORRESPONDING TO THE SITUATION IN FIG.1

\begin{tabular}{|c|c|c|c|c|c|}
\hline & Rpt 0 & Rpt 1 & Rpt2 & Rpt 3 & no-assoc. \\
\hline track 0 & 0 & 0.273 & 0 & 0 & 0.727 \\
\hline track 1 & 0 & 0 & 0.156 & 0.779 & 0.064 \\
\hline track 2 & 0 & 0 & 0.651 & 0.240 & 0.109 \\
\hline track 3 & 0 & 0 & 0 & 0 & 1 \\
\hline track 4 & 0.955 & 0 & 0 & 0 & 0.045 \\
\hline track 5 & 0 & 0.694 & 0 & 0 & 0.305 \\
\hline
\end{tabular}

However, during the experiments, we also noticed that the JPDA algorithm may sometimes yield poor performance 
when the observations are compactly located. In this situation, the weighted-average method of track updating will lead to biased tracks, because any track on a particular target will be attracted, to some extent, toward any neighboring target with which it correlated consistently. This is often referred to as the track coalescence problem [18]. The solution here is to employ a nearest neighbor version of JPDA, i.e., the association likelihood is calculated jointly as in the original JPDA, while the track update is not based on a weighted sum of all the available reports, but rather the one with highest likelihood.

\section{EXPERIMENTS}

The approached described in the paper has been tested on a video sequence taken from the European project CAVIAR. The video sequence is taken from a shopping center in Portugal. An example is shown in the pictures of Fig.2 and Fig.3. The data sequence from CAVIAR gives annotated ground truths for the detection of the pedestrians (marked as red rectangles). Based on this given data, the uncertainties and occlusions have been simulated. This simulated data is then applied to the implementation described in this paper. In this section, we show experimentally that it is possible to recover objects from the BOF grids and perform tracking at the object level although the notion of an object does not exist in the BOF grids.

The results shown in Fig.2 and Fig. 3 is shown in its time sequence. For each time instance, the left figure shows the input image with the bounding boxes, each indicating the detection of a human, after the simulation of uncertainties and occlusions. The lower figure shows the corresponding visualization of the bayesian occupancy filter. The color intensity of the cells represents the occupation probability of the cell proportionally. The little arrows in a cell gives the average velocity of the velocity distribution for the cell. The right figure depicts the tracker output given by the NNJPDA tracker with the numbers indicating its track number. The sensor model used is a 2D planar gaussian model projected on the ground. Its coordinates are given by the lower edge of the bounding box.

The characteristics of the BOF can be inferred from Fig.2 and Fig. 3. A diminished occupancy of the person further away from the camera is seen from the data between the first and second step. This is caused by occasional instability in the human detection. The occupancy in the BOF grids for the missed detection diminishes gracefully with time rather than disappearing immediately for classical occupation grids. This mechanism provides a form of temporal smoothing to handle unstable detections.

A more challenging occlusion sequence is shown in the last three rows of Fig.2 and Fig. 3. Due to a relatively longer period of occlusion, the occupancy probability of the occluded person becomes weak. However, with an appropriately designed tracker such as in our implementation, these problems can be handled at the object tracker level. The tracker manages to
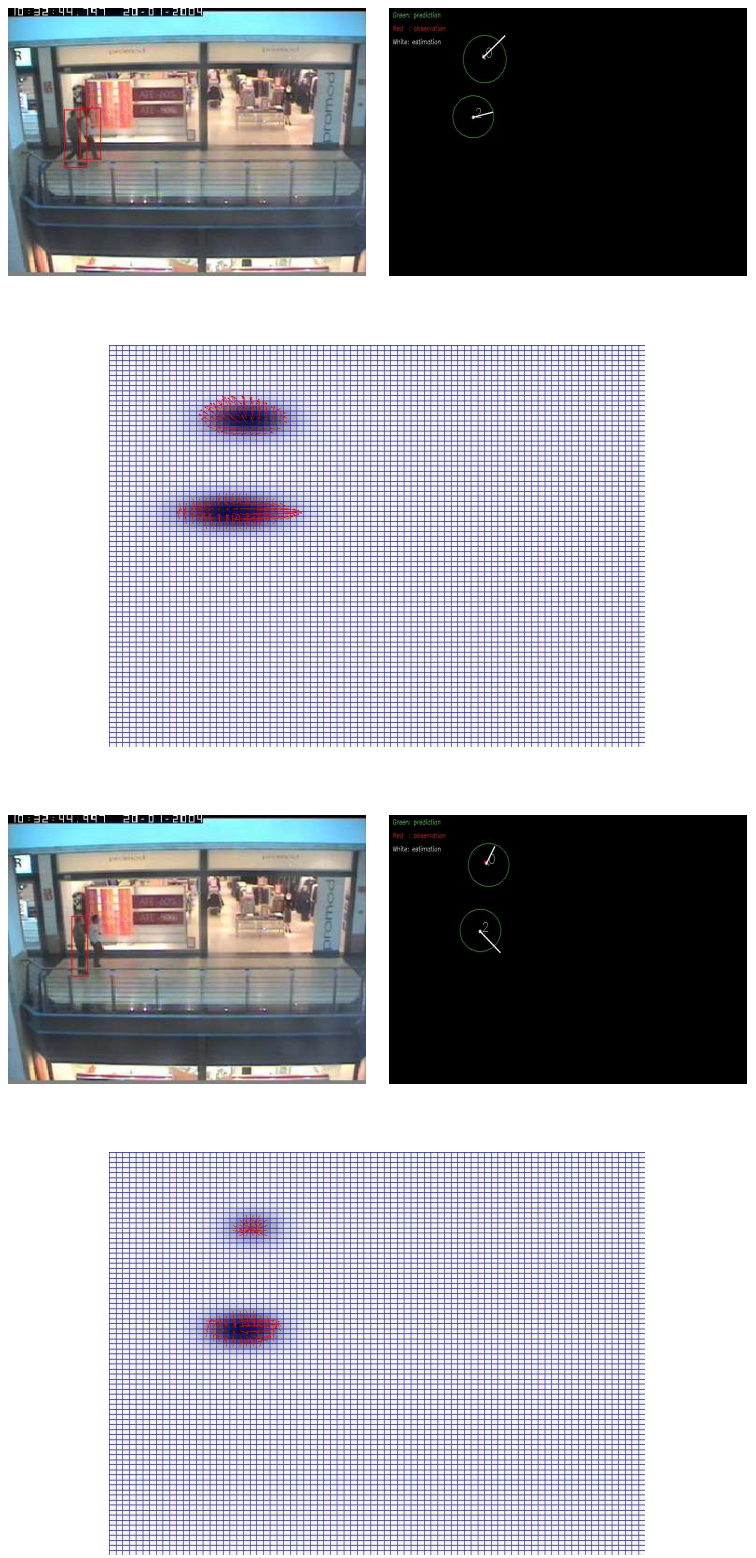

Fig. 2. Caviar data and results. To be continued in Fig.3.

track the occlusion at the object level, as shown in the last step.

\section{CONCLUSION}

This paper elaborated the theory and implementation of Bayesian occupancy filter (BOF), which is a gridmap based approach to model dynamic environment. This probabilistic framework is developed based on Bayesian theory. It can accurately estimate the geometric state (occupancy) and dynamic state (the motion) of the environment. In this work, BOF is applied to a multiple-object tracking problem and satisfactory results have been demonstrated.

For some specific applications, in this work, we further abstract the BOF onto the object level. An object extraction 
algorithm is implemented to segment the occupied regions from the estimated gridmap. Each region is then handled as an object by the classic Kalman filter. To resolve the ambiguity in data association. A Nearest Neighborhood JPDA algorithm is included and tested.

\section{REFERENCES}

[1] C. Coué, T. Fraichard, P. Bessiere, and E. Mazer, "Multi-sensor data fusion using bayesian programming: an automotive application," in Proc. Int'l. Conf. Intelligent Robots and Systems, 2002.

[2] C. Coue, T. Fraichard, P. Bessiere, and E. Mazer, "Using bayesian programming for multi-sensor multitarget tracking in automotive applications," in Proc. Int'l. Conf. Robotics and Automation, 2003.

[3] K. Mekhnacha and E. M. an P. Bessiere, "The design and implementation of a bayesian cad modeler for robotic applications," Advanced Robotics, vol. 15, no. 1, pp. 45-70, 2001.

[4] S. Thrun, W. Burgard, and D. Fox, Probabilistic Robotics. MIT Press, 2005.

[5] K. O. Arras, N. Tomatis, and R. Siegwart, "Multisensor on-the-fly localization: precision and reliability for applications," Robotics and Autonomous Systems, vol. 34, pp. 131-143, 2001.

[6] A. H. Jazwinsky, Stochastic processes and filtering theory. New York: Academic Press, 1970.

[7] Y. Bar-shalom and W. D. Blair, Multitarget-multisensor tracking: application and advances. Boston: Artech House, 2000, vol. 3.

[8] H. Gauvrit, J. P. L. Cadre, and C. Jauffret, "A formulation of multitargets tracking as an incomplete data problem," IEEE Trans. Aerospace and Electronic Systems, vol. 33, no. 4, pp. 1242-1257, 1997.

[9] S. S. Blackman and R.Popoli, Design and analysis of modern tracking systems. Norwood,MA: Artech House, 1999.

[10] C.-C. Wang, C. Thorpe, and S. Thrun, "Online simultaneous localization and mapping with detection and tracking of moving objects: theory and results from a ground vehicle in crowded urban areas," in Proc. Int'l. Conf. Robotics and Automation, September 2003, pp. 842-849.

[11] H. Moravec, "Sensor fusion in certainty grids for mobile robots," $A I$ Magazine, vol. 9, pp. 61-74, 1988.

[12] A. Elfes, "Using occupancy grids for mobile robot perception and navigation," Computer, vol. 22, no. 6, pp. 46-57, 1989.

[13] E. Prassler, J. Scholz, and A. Elfes, "Tracking multiple moving objects for real-time robot navigation," Journal of Autonomous Robots, Special Issue on Perception for Mobile Agents, vol. 8, 2000.

[14] R. E. Kalman, "A new approach to linear filtering and prediction problems," Journal of Basic Engineering, vol. 35, 1960

[15] P. F. Felzenszwalb and D. P. Huttenlocher, "Pictorial structures for object recognition," International Journal of Computer Vision, no. 1, pp. 5579, 2005.

[16] D. Schulz, W. Burgard, D. Fox, and A. B. Cremers, "People tracking with a mobile robot using sample-based Joint Probabilistic Data Association Filters," International Journal of Robotics Research, no. 2, pp. 99-116, 2003.

[17] C. Rasmussen and G. D. Hager, "Probabilistic data association methods for tracking complex visual objects," IEEE Trans. Pattern analysis and machine intelligence, vol. 23, no. 6, pp. 560-576, 2001.

[18] J. A. Roecker and G. L. Phillis, "Suboptimal joint probabilistic data association," IEEE Trans. Aerospace and Electronic Systems, vol. 29 no. 2 , pp. $510-517,1993$
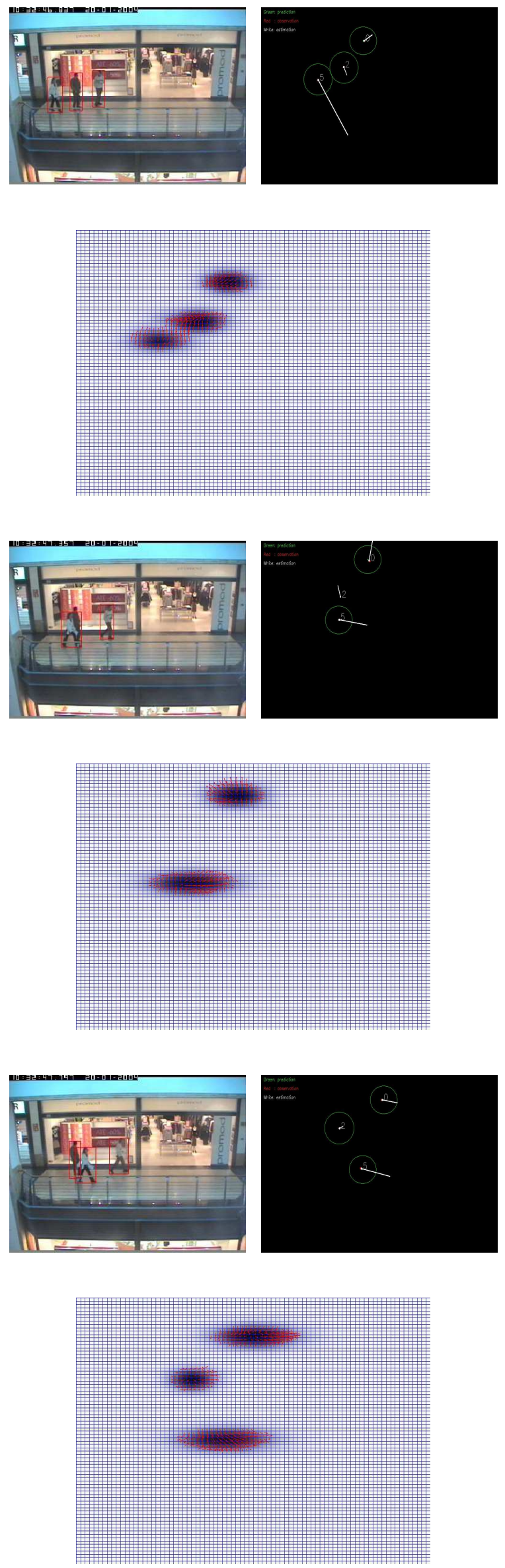

Fig. 3. Together with Fig.2. Tracking sequence based on the data from CAVIAR. The first column shows the input image with the bounding boxes indicating detected humans. Second column and third column shows the corresponding representation of the BOF and JPDA tracker respectively. 